\title{
Absence of Weight Regulation in Exercising Hamsters
}

\author{
KATARINA TOMLJENOVIĆ BORER \\ Neuroscience Laboratory, University of Michigan, Ann Arbor, Michigan 48104
}

(Received 9 October 1973)

\begin{abstract}
BORER, K. T. Absence of weight regulation in exercising hamsters. PHYSIOL. BEHAV. 12(4) 589-597, 1974. Spontaneous activity on horizontal discs causes a permanent upward displacement of hamster weight, length, and, under some circumstances, percentage body fat. This phenomenon manifests itself through an immediate increase in the rate of weight gain, a disappearance of a preference for sunflower seeds, as well as in a delayed and gradual increase in food intake during and immediately following the activity period. A week after termination of spontaneous disc running normal regulation of body weight is reinstated and maintained at the new elevated weight level. These results suggest that some concomitant of spontaneous disc running in hamsters renders the weight-regulatory mechanisms inoperative.
\end{abstract}

Spontaneous exercise Weight gain Growth Body fat Food consumption Food selection

Weight regulation

MAINTENANCE of constant body weight and body composition against the environmental challenges of variable temperature and food availability is one of the more remarkable features of homeotherms. The least understood aspect of the weight-regulatory mechanism is the identity of signals that are received and utilized by the brain for the maintenance of weight constancy. The widely accepted lipostatic.view of weight regulation proposes that either the absolute levels of lipid reserves $[9,14]$ or the amount of nocturnal fat deposition [16] is the monitored variable but does not operationally define the agent transmitting the relevant information to the nervous system.

One of the obstacles to elucidation of processes involved in weight-regulatory mechanisms is scarcity of laboratory manipulations which can permanently obstruct weight regulation in a neurologically and hormonally intact animal. For instance, the displacements of body weight in adult rats above or below their regulated level by force-feeding [5], food restriction [25], or exercise [3], stimulate compensatory changes in the levels of feeding, metabolism, and activity which restore the body weight to its premanipulation level once the environmental challenges are removed.

We report in this article a simple procedure which appears to interfere with the process of weight regulation in adult hamsters. Voluntary activity on horizontal disc exercisers accelerates the rate of weight gain in hamsters and causes a permanent upward displacement of their body weight, body length, and, under some circum- stances, percentage body fat. Weight regulation is restored to its pretreatment level several days after termination of voluntary exercise. This preparation could serve as a valuable model in elucidation of signals and processes operating in weight-regulatory mechanism in the hamster.

\section{METHOD}

Animals

Fifty-six mature golden hamsters (Con Olson, Madison, Wisconsin), weighing on the average. over $100 \mathrm{~g}$ at the beginning of experiments, were used. Twenty-four female hamsters were employed in the main experiment which took place from April to June. The experiment was replicated with 32 other hamsters, 24 females and eight males between January and April.

\section{General Procedure}

Figure 1 outlines the experimental design. Four groups of six hamsters were matched by weight and, in the experimental replications, also by sex. Two groups, each, were assigned to sedentary condition and exercise manipulation, respectively. One of the two sedentary and exercise groups was provided ad lib with Ralston Purina laboratory chow pellets throughout the experiment. The other dietary condition was assigned to the remaining sedentary and exercise groups. These animals received unlimited amounts of sunflower seeds in $7 \mathrm{~cm}$ high, $7 \mathrm{~cm}$ wide steel cups as well as pellets. All hamsters were

\footnotetext{
${ }^{1}$ This research was supported by NIMH Grant No. MH20811 and NASA Grant No. NGR 23-005-487 to E. S. Valenstein. The author gratefully acknowledges the encouragement and support of Elliot $S$. Valenstein and the technical assistance of Louise Braisted and Patricia Britten. Elliot S. Valenstein, Charlotte Mistretta and J. Bradley Powers critically read an earlier draft of this paper.
} 


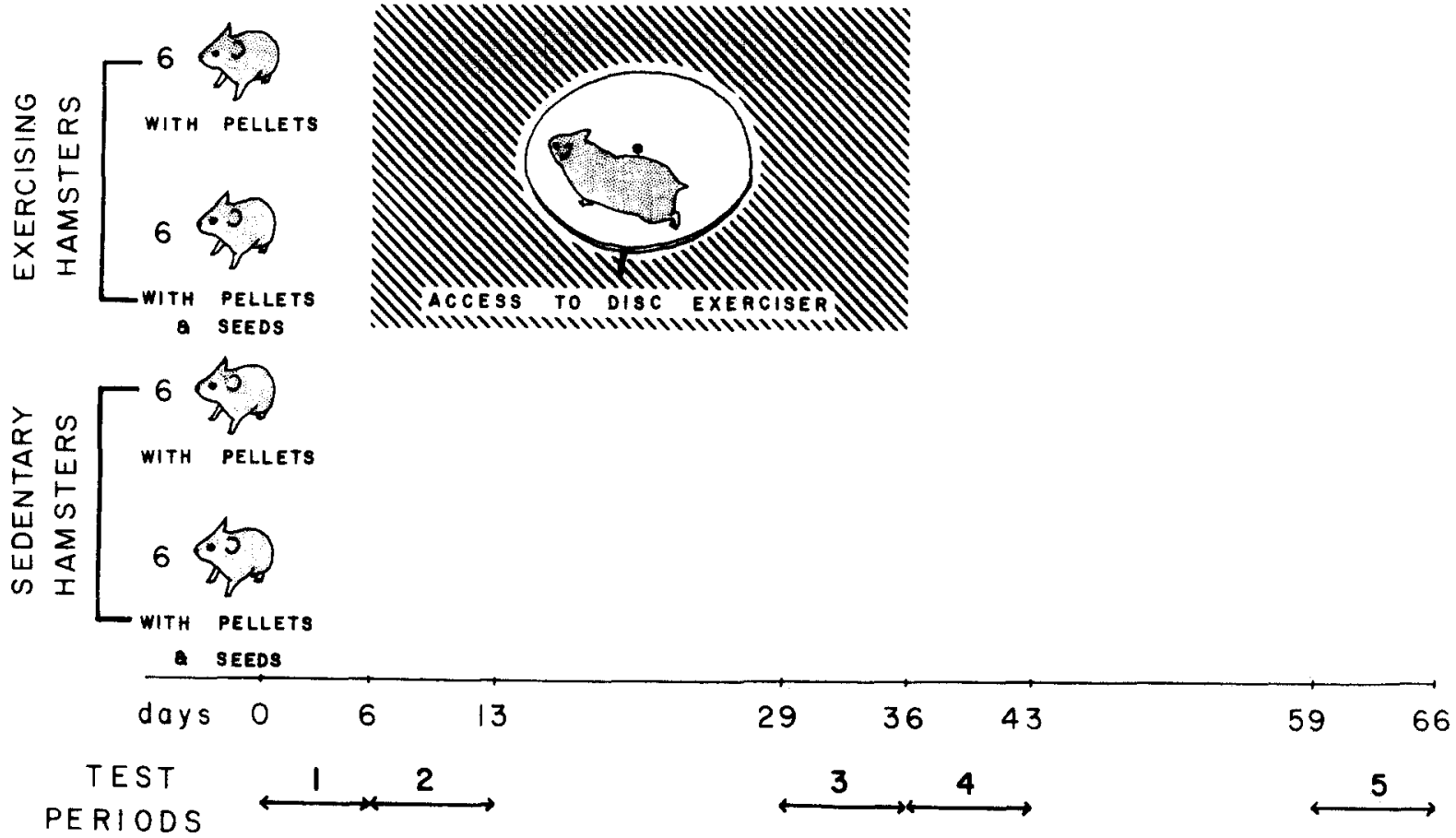

FIG. 1. A diagram illustrating the general experimental procedure. (See method section for details.)

housed in the same room on a reversed 12L:12D light cycle. The maintenance activities and the various measurements were performed daily before the start of the dark cycle. Sedentary hamsters were individually housed in suspended cages at all times. Exercising hamsters also occupied suspended cages except for the duration of voluntary activity, at which time they were individually housed in Plexiglas boxes $(30 \mathrm{~cm}$ wide, $40 \mathrm{~cm}$ deep and $32 \mathrm{~cm}$ high) with a permanently mounted horizontal disc exerciser, $25 \mathrm{~cm}$ in diameter $[1,11]$.

The hamsters were allowed to adjust to the dietary options for seven days. After this, the two exercise groups were placed into boxes with disc exercisers for 30 days. After about four weeks of access to activity discs, exercising hamsters were retired to suspended cages for an additional four-week period.

All hamsters were weighed daily. The activity levels of exercising hamsters were recorded daily on cumulative counters which were activated by full revolutions of individual discs. Because of the effort involved in measuring food and sunflower seed spillage, the metsurements of food and water intake were taken only during five discrete experimental periods, each lasting seven days (Fig. 1): (1) immediately prior to access to disc exercisers, (2) during the first week of exercise, (3) during the last week of exercise, (4) immediately after retirement from disc exercisers, and (5) during the fourth week after termination of exercise.

The caloric content of pellets was taken to be 3.61 $\mathrm{Cal} / \mathrm{g}[2,23]$. This value was obtained by applying the established yields of $4 \mathrm{Cal} / \mathrm{g}$ for protein and carbohydrate and $9.3 \mathrm{Cal} / \mathrm{g}$ for fat to Ralston Purina chow which contains $23.4 \%$ protein, $55 \%$ carbohydrate, and $4.3 \%$ fat. The same procedure was followed in establishing the caloric content of $6.83 \mathrm{Cal} / \mathrm{g}$ for sunflower seed kernels which contain $23.5 \%$ protein, $9.7 \%$ carbohydrate, and $61 \%$ oil [ 6 , $13,17]$

In the two replications of the experiment the same procedure was followed except that food and water measurements were omitted.

On the last day of the experiment measurements were taken of the hamster wet weight and of the snoutto-tail-tip length. Two measures of adiposity were taken at that time from the exercised and sedentary hamsters: the percentage body fat content and the weight of the limb subcutaneous fat depots. Total body fat was determined indirectly from the total body water content of hamsters which bears a constant relationship of $73.2 \%$ to the lean body mass $[15,18]$. The body water content was determined after drying carcasses to a constant weight at $90-105^{\circ} \mathrm{F}$ for several days. In a different group of hamsters, prominent subcutaneous fat pads were exercised bilaterally from the underside of front and rear limbs, rinsed in saline, blotted on filter paper and weighed.

Measurements were also made of the adrenal weight. The adrenal glands were excised bilaterally, blotted dry on filter paper, and weighed on an analytical balance.

The analysis of between-group data was done with Duncan's multiple range test, and the within-group comparisons were made with Wilcoxon's matched pairs signed ranks test (two tailed).

\section{RESULTS}

\section{Body Weight Changes}

Figure 2 illustrates the weight changes induced by the dietary and exercise variables. The addition of sunflower seeds to the standard diet produced a 1.6 fold 


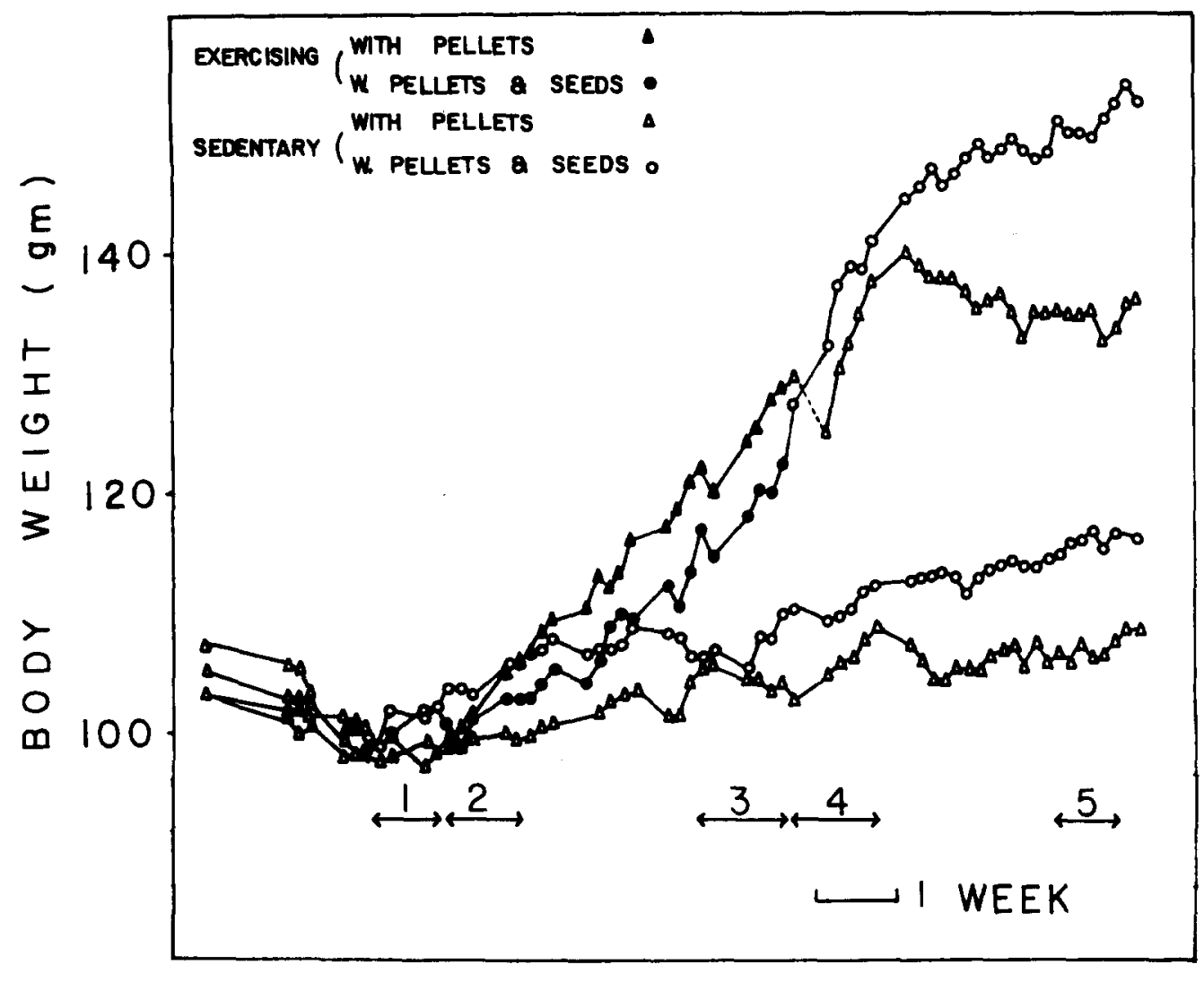

$T \perp M E$

FIG. 2. Body weight changes of the main group as a function of diets and exercise on horizontal discs. Solid symbols mark weight during exercise, open symbols: sedentary weight, triangles: weight on a diet of pellets, circles: weight on a diet of pellets and seeds.

increase in the rate of weight gain to $0.2 \mathrm{~g} /$ day in sedentary hamsters. However, the weight difference between the sedentary hamsters with pellets and seeds (open circles) and hamsters with pellets only (open triangles) did not reach significance by the last day of the experiment.

By contrast, voluntary running on disc exercisers (Fig. 2, solid symbols) exerted a pronounced influence over the rate of weight gain in hamsters. For the duration of access to disc exercisers this group of hamsters gained weight at the rate of $0.8 \mathrm{~g} /$ day. The increased rate of weight gain was largely independent of dietary influences, although the pellets group at $0.9 \mathrm{~g} /$ day, slightly outgained the pellets-and-seeds group, at $0.7 \mathrm{~g} / \mathrm{day}$. The difference between the mean body weight of exercising and sedentary hamsters reached statistical significance $(p<0.01)$ during the last seven days of running.

After the termination of exercise a transient, but even more pronounced, spurt of weight gain took place in all of the retired hamsters (Fig. 2). The magnitude of change in the rate of weight gain, in the final weight level attained, and the duration of the weight-gain spurt, were all strongly influenced by the dietary variable. Immediately upon the retirement from disc exercisers hamsters with pellets and seeds gained $2.1 \mathrm{~g} /$ day for 11 days while hamsters with pellets only accumulated $1.5 \mathrm{~g} /$ day for 10 days. After the spurt of weight gain associated with the termination of exercise subsided, hamsters with pellets and seeds resumed gaining weight at the original rate characteristic of sedentary hamsters with this dietary option. The retired hamsters with access to pellets lost some of the accumulated weight at the rate of $0.6 \mathrm{~g} / \mathrm{day}$ during the next seven days before their weight stabilized at the somewhat lower level. The divergent weight changes, influenced by the dietary options of retired hamsters, generated a significant $16 \mathrm{~g}$ weight difference $(p<0.05)$ between the dietary groups by the last week of the experiment.

The exercise-induced increase in the weight gain was replicated with two other groups of hamsters. A group of 10 hamsters increased their rate of weight gain from $0.1 \mathrm{~g} /$ day or less to $1.0 \mathrm{~g} /$ day during the 26 -day access to disc exerciser. After the termination of activity the four retired hamsters with pellets and seeds accumulated 2.0 g/day for 10 days before resuming their original sedentary rate of weight gain, while the six retired hamsters with pellets only accumulated $1.8 \mathrm{~g} /$ day for 9 days before losing some weight and levelling off $19 \mathrm{~g}$ below the body weight of pellets-and-seeds group. Similar course of weight changes took place during the second replication of this phenomenon with a group of twelve hamsters except that the disc-induced rise in the rate of weight gain did not exceed $0.5 \mathrm{~g} /$ day and that the retirement weight-gain spurt was followed by slight weight losses in both dietary groups. During both replications the dietary influences 


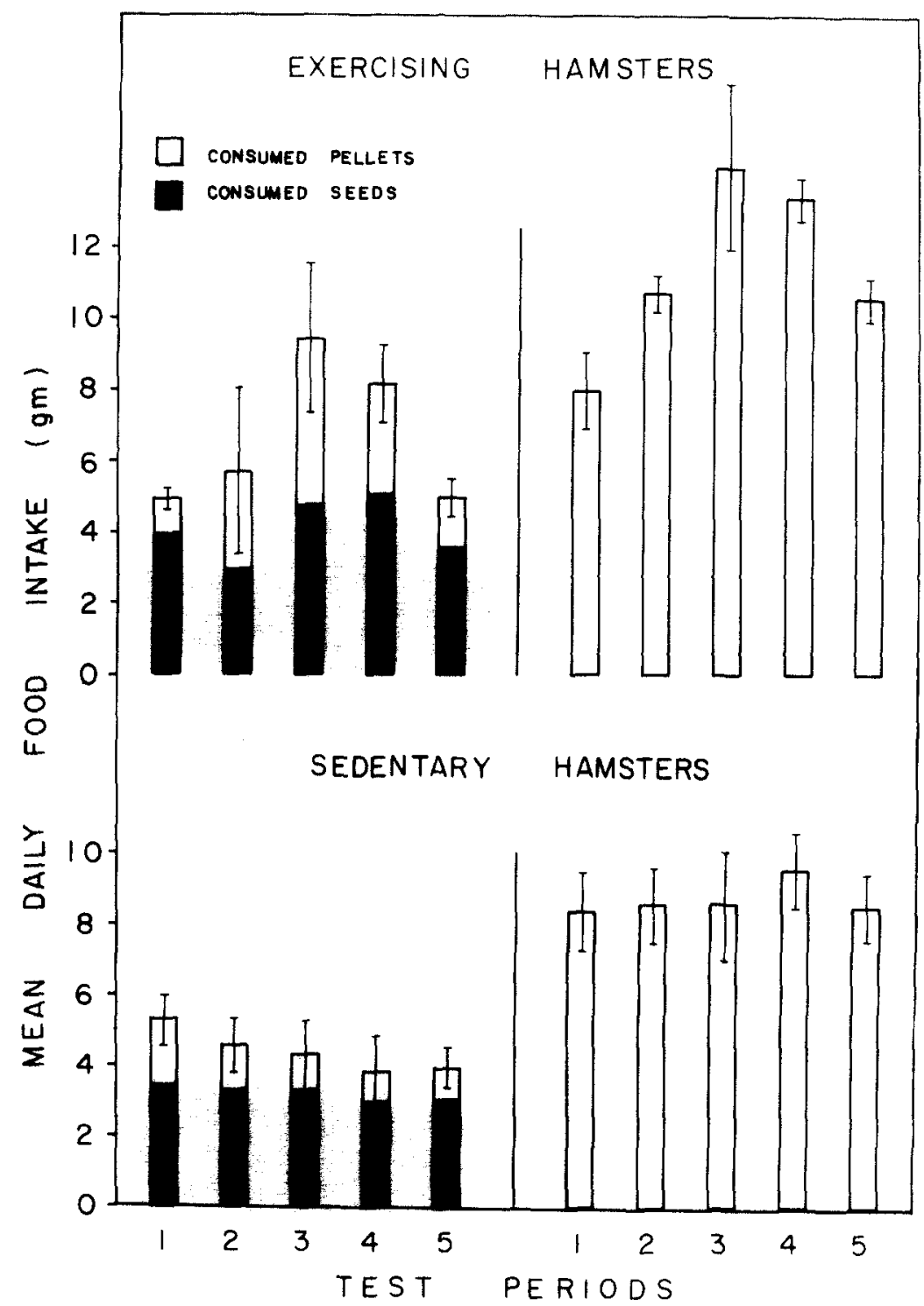

FIG. 3. Mean daily food intake, in grams of pellets (open bars) or of sunflower seed kernels (solid bars), of exercising and sedentary hamsters during the five seven-day test periods.

over the weight gain of sedentary hamsters were similar to those seen in the main experiment.

In spite of the small number of males employed in this study, there were apparent sex differences in the magnitude of exercise-induced weight gain. Four females matched by weight at the start of the experiment to four males $(107 \mathrm{~g})$ gained $25.8 \pm 11.7$ during the four weeks of disc running. Unlike the uniform response by females, only two males exhibited an accelerated weight gain during the four weeks of running. The $19.5 \mathrm{~g}$ difference between the exerciseinduced weight gain of females and males was reliable at $p<0.05$. The weight-gain spurt after the termination of exercise appeared in all animals and was of the same magnitude in males and in females (14.3 and $15.5 \mathrm{~g}$, respectively).

\section{Activity Levels on Disc Exercisers}

Hamsters generated an average of between 15,000 and 35,000 disc revolutions per day, but no clear pattern of running over time or with respect to diet emerged. At high levels of running (over 40,000 revolutions per day) some hamsters developed blisters on the paws which left a fine spray of blood over the wheel and edges of cage. There were no sex differences in activity levels. The four males and four females which differed in the magnitude of weight-gain increase during exercise, generated an average of 25,047 and 26,200 disc revolutions per day, respectively. Spearman rank coefficient of correlation between the mean daily running levels of hamsters in all three exercising groups and the maximal weight gain associated with the exercise was 0.25 , indicating that only about 6 percent of the variability in the weight change could be attributed to variation in activity levels generated by individual hamsters.

\section{Changes in the Quantity of Food Eaten}

The amounts of pellets (open bars) and of sunflower seeds (solid bars) eaten by hamsters under sedentary and 


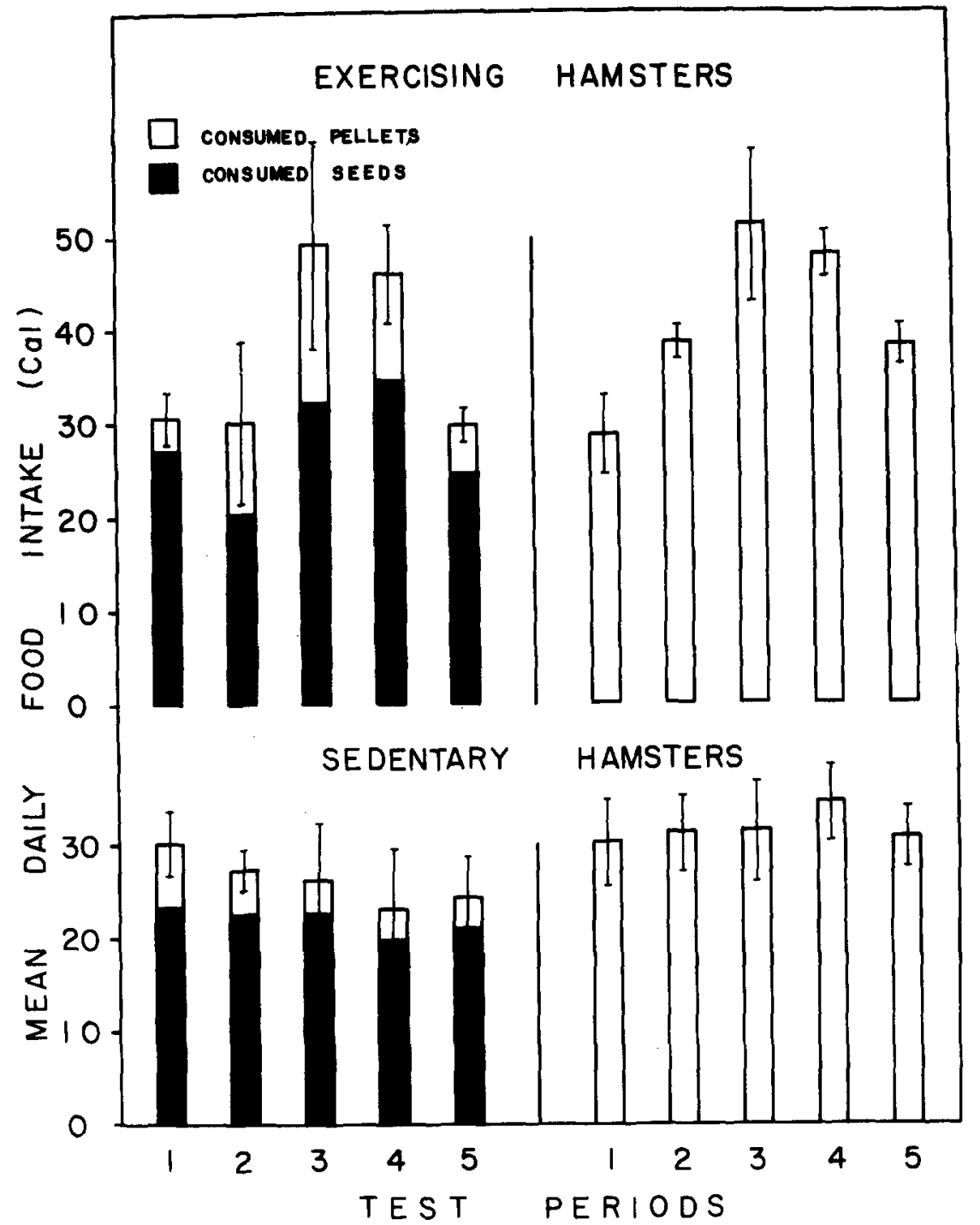

FIG. 4. Mean daily food intake, in Calories from pellets (open bars) or from sunflower seed kernels (solid bars), of exercising and sedentary hamsters during the five seven-day test periods.

exercising conditions are presented in gram units in Fig. 3 and in Calorie units in Fig. 4.

Sedentary hamsters with pellets only ingested daily $8-9 \mathrm{~g}$ of pellets (Fig. 3, lower right quadrant) or 30 Calories (Fig. 4) and maintained a weight gain of $0.1 \mathrm{~g} / \mathrm{day}$ throughout the nine-week experimental period. Sedentary hamsters with a choice of pellets and seeds started out with the identical intake of $30 \mathrm{Cal} / \mathrm{day}$ or $5.3 \mathrm{~g}$ of food but reduced it by $22 \%$ by the fifth week of the experiment. In spite of this decreased caloric intake, they gained weight 1.6 times faster than the hamsters restricted to a diet of pellets.

Exercise did not affect the caloric intake of hamsters with a choice of pellets and seeds during the first week of running (Fig. 4, upper left quadrant). By contrast, hamsters restricted to pellets ate $34 \%$ more food during the first week of running $(p<0.05)$. By the last week of running the caloric intake of both dietary groups had reached an equally high level of about 50 Calories per day (Fig. 4 , upper half). This represented a significant increase above the baseline sedentary intake $(p<0.05)$. During the first seven days of retirement from disc exercisers the hamsters continued to ingest the large caloric intakes reached in the course of four weeks of voluntary running, 48 and 46 $\mathrm{Cal} / \mathrm{day}$ for the groups with pellets and pellets and seeds, respectively. By the last experimental period the food intake of hamsters with pellets and seeds had returned to the normal sedentary level, while the food intake of hamsters with pellets remained 33 percent above their sedentary baseline $(p<0.05)$.

\section{Changes in Dietary Selection}

Sedentary hamsters displayed small gradual shifts in dietary selection in the course of the experiment (Fig. 3 and 4). They progressively reduced their intake of pellets from 1.9 to $0.9 \mathrm{~g} /$ day by the fifth week of the experiment with the concomitant increase in the preference for sunflower seeds from $64 \%$ to $77 \%$. 
An immediate change in dietary selection was associated with the onset of voluntary disc running (Fig. 3, upper left quadrant). Throughout the period of spontaneous exercise hamsters abandoned a sedentary preference of $80 \%$ for sunflower seeds over pellets for an almost equivalent intake of pellets $(48 \%)$ and seeds $(52 \%)$. During the first seven days of retirement from exercise the hamsters continued to show a lower preference for sunflower seeds $(62 \%)$ than they did during the original sedentary period (1) or during the final sedentary period (5) (72\%).

\section{Changes in Fluid Intake}

There were no changes in fluid intake that could be attributed to exercise. However, a diet of pellets required higher levels of fluid intake $(14-17 \mathrm{ml} /$ day $)$ than a diet of pellets and seeds which was associated with as low an intake of water as $7-9 \mathrm{ml} /$ day.

\section{Changes in Body Length and Composition}

The changes in body length and in the several indices of body adiposity under the influence of diets and exercise are presented in Table 1. Thirty-three days after retirement from disc exercisers the hamsters in the exercised groups were significantly longer $(p<0.05)$ than the hamsters in sedentary groups. The difference in length was greater $(p<0.01)$ between the retired and sedentary hamsters with pellets only than between the corresponding groups eating pellets and seeds.

Hamsters with a choice of pellets and seeds contained a larger percentage of fat in their bodies than the hamsters with pellets in either the exercised groups $(23.3 \mathrm{vs} 13 \%$, respectively, $p<0.01)$ or the sedentary groups $(16.5 \mathrm{vs}$ $11.6 \%$, respectively, $p<0.05)$, and the activity enhanced this difference.

The subcutaneous fat pads constituted a significantly greater percentage of the total body weight $(6.4 \%)$ in retired hamsters with pellets and seeds than in both of the sedentary groups $(p<0.01)$. The fat pads of retired hamsters with pellets did not differ from those of hamsters in the two sedentary groups.

As it became obvious that a regime of voluntary exercise was associated with both a change in length as well as with a change in percentage body fat, an obesity index was computed as a ratio of percentage body fat to body length to assess the relative magnitude of change in these two variables. In spite of significant length differences between the exercising and sedentary groups with pellets, there was no difference between their obesity indices which were quite low at 0.7 . By contrast, sedentary hamsters with pellets and seeds had a $36 \%$ higher obesity index of $0.95(p<0.05)$, and the retired hamsters with pellets and seeds had an $84 \%$ higher obesity index of $1.29(p<0.01)$.

\section{Changes in Adrenal Weight}

Both exercise and dietary enrichment caused an increase in the wet weight of adrenals relative to body weight, but only the former variable produced significant differences $(19.3 \pm 3.5 \mathrm{mg} / 100 \mathrm{~g}$ in exercised group with seeds vs. $14.9 \pm 2.4$ and $12.8 \pm 3.5 \mathrm{mg} / 100 \mathrm{~g}$ body weight in the two sedentary groups, $p<0.05$ ).

TABLE 1

DIFFERENCES IN BODY LENGTH AND BODY COMPOSITION OF HAMSTERS FIVE WEEKS AFTER RETIREMENT FROM DISC EXERCISERS

\begin{tabular}{lcccc}
\hline & \multicolumn{2}{c}{ EXERCISED HAMSTERS } & \multicolumn{2}{c}{ SEDENTARY HAMSTERS } \\
& Pellets and seeds & Pellets only & Pellets and seeds & Pellets only \\
& $\mathrm{n}=6(154.5 \mathrm{gm})^{*}$ & $\mathrm{n}=6(135.6 \mathrm{gm})$ & $\mathrm{n}=6(117.6 \mathrm{gm})$ & $\mathrm{n}=6(108.5 \mathrm{gm})$ \\
& $18.07 \pm 0.4 \dagger$ & $18.12 \pm 0.2 \ddagger$ & $17.37 \pm 0.3$ & $17.02 \pm 0.6$ \\
$\begin{array}{l}\text { Body length (cm) } \\
\begin{array}{l}\text { Percentage } \\
\text { body fat }\end{array}\end{array}$ & $23.26 \pm 1.4 \S$ & $12.99 \pm 2.3$ & $16.48 \pm 3.5 \dagger$ & $11.62 \pm 4.5$ \\
$\begin{array}{l}\text { Obesity index } \\
\text { (percentage body } \\
\text { fat/body length) }\end{array}$ & $1.29 \pm 0.1 \S$ & $0.72 \pm 0.1$ & $0.95 \pm 0.2 \pm$ & $0.68 \pm 0.3$ \\
& $\mathrm{n}=6(141.3 \mathrm{gm})$ & $\mathrm{n}=6(127.3 \mathrm{gm})$ & $\mathrm{n}=5(120.9 \mathrm{gm})$ & $\mathrm{n}=5(113.3 \mathrm{gm})$ \\
$\begin{array}{l}\text { Fat pad weight } \\
\text { (gm/100 gm } \\
\text { body weight })\end{array}$ & $6.4 \pm 1.9 \S$ & $4.9 \pm 1.7$ & $3.8 \pm 0.4$ & $3.2 \pm 1.0$ \\
\hline
\end{tabular}

*Numbers in parentheses refer to the mean body weights of hamster groups on the day of measurements.

$\dagger p<0.05$ significantly greater than the corresponding sedentary group.

$\ddagger p<0.01$ significantly greater than the corresponding sedentary group.

$\S p<0.01$ significantly greater than the other three groups.

$a p<0.05$ significantly greater than the two groups restricted to pellets only. 


\section{DISCUSSION}

Weight regulation in the adult hamster manifests itself through maintenance of steady body-weight levels or low rates of weight gain over long periods of time. It is also evident in the constant caloric intake which hamsters maintain when given diets differing in caloric density. This weight regulation appears disrupted when hamsters engage in voluntary activity on horizontal disc exercisers. During voluntary activity there is a great acceleration in the rate of weight gain. The upward displacements of body weight, generated by rapid weight gain over several weeks of activity, appear permanent, and the normal regulation of body weight is restored at the new weight plateau some days after the cessation of running. The weight displacements produced by disc running stand in marked contrast to such displacements produced by force-feeding, food restriction, or activity in vertical wheels. The latter procedures produce only transient and reversible changes in body weight ([ 5 , 25 , and 3 , respectively), and the original body weight is restored through the regulatory changes in feeding behavior, metabolism, and activity once the environmental constraint is removed. Disc running appears to obstruct the mechanism that maintains some record of the original body weight during weight displacements. No compensatory changes toward restoring the original body weight are apparent after termination of disc running. Instead, weight regulation resumes after cessation of exercise as if no displacements of body weight took place.

A number of changes related to weight and food intake took place during or immediately after disc exercise in hamsters. The changes that were clearly directly related to running were an increase in the rate of weight gain, a change in dietary selection patterns, and growth in length.

Two features of exercise-induced weight gain stand out. One is the steady maintenance of the high weight gain throughout the period of activity. One could prolong the exposure of hamsters to activity discs to determine the physical limits to further weight increase. This technique could also be used to determine whether some homeostatic process exists which would prevent weight increases above some maximal body size analogous to mechanisms operating in the rat which defend a minimal body weight against estrogen-mediated weight losses [26].

Changes in dietary selection followed the same time course and maintained the same relationship to running as the weight changes. If this indicates a causal relationship it would be of value to identify the agent responsible for both the weight gain and preference change to establish the functional significance of a dietary shift from a high-fat, lowcarbohydrate diet during inactivity to an equivalent intake of protein, fat, and carbohydrate during disc running. Increased selection of carbohydrate was reported in other energy-expending situations such as running in vertical wheels [4]. On the other hand, the absence of marked preferences for palatable substances is also characteristic of prepuberal growth [24]. It would also be necessary to determine that the observed shift in dietary selection reflects altered nutritional needs rather than response competition between running and seed shelling.

The second striking feature of disc activity was that it affected differentially the weight gain in males, females, and the three individual groups of hamsters although they all engaged in similar levels of running. In the same vein, there was little correlation between the amount of running by individual hamsters and the magnitude of induced weight gain. This characteristic of exercise-induced weight gain suggests that spontaneous disc running stimulates events which are sensitive to influences of gonadal hormones, age, and possibly season.

A number of features of exercise-induced weight gain are compatible with the hypothesis that activity on horizontal disc exercisers stimulates the secretion of growth hormone by the pituitary. Of the four groups of hamsters which were initially matched by weight, only the exercised hamsters displayed significantly longer carcasses at the end of the experiment. Physical exercise is a known stimulus for a transient increase of growth hormone relase in man [8, 12, 22], and estrogen has been shown to potentiate this response [7]. Operation of a similar process in the hamster would account for the observed sex differences in weight gain during activity on discs, even though under sedentary circumstances estrogen and testosterone exert little influence over the hamster body weight [28]. Age differences may have been responsible for the differential effectiveness of disc exercise in stimulating a weight gain. Hamsters were obtained from the breeder by requesting adult animals of maximal size rather than specifying an age group. Experiment was conducted over a 6 month period, and possible seasonal influences can not be excluded.

It is not clear which feature of activity on horizontal disc is responsible for accelerated weight gain and alterations in feeding behavior. Exercise may not be the effective stimulus in this situation despite its established role in growth hormone secretion. Activity in the vertical wheels by hamsters and other small animals has quite the opposite effect on body weight and ingestive behavior to that observed on horizontal discs. There is an initial period of decreased feeding and a weight loss which is not compensated for throughout the period of running in vertical wheels, although both feeding and weight regulation soon return to normal [3]. Both activity devices require energy for their activation, but hamsters may have to work harder in vertical wheels to counteract the stalling action of gravity than to set discs to spin. This difference may partly explain two to four times higher activity levels of hamsters on discs compared to hamsters in wheels [27].

Hamsters with access to discs can be observed to take free rides once the centrifugal force of the disc is generated by a burst of running activity, and these free rides may account for some undetermined portion of total disc revolutions. The free-ride feature of activity on discs may well be the effective stimulus to increased weight gain and alterations in feeding. It is possible that spinning on a horizontal disc is more stimulating, arousing, and reinforcing than running in a vertical wheel. Lack of stimulation and arousal is known to suppress growth and growth hormone release $[19,20]$, and increased stimulation and arousal have been associated with growth-hormone secretion and catch-up growth $[19,20,21]$. Thus both physical activity and arousal associated with running on a disc may contribute to observed weight and feeding changes in the hamster.

The increased caloric intake appeared to be secondary to changes in the rate of weight gain. It appeared with a delay of several days in the pellets-and-seeds group, and it developed gradually to reach a maximum of about 50 calories per day during the last week of exercise. The magnitude of this increase in food intake is remarkable in view of difficulties in detecting such increases in hamsters under other circumstances of energy expenditure such as pregnancy 
[28]. The persistence of this hyperphagia for several days after termination of activity may be largely responsible for the retirement weight-gain spurt and for the exaggerated adiposity found in the pellets-and-seeds hamsters at the end of the experiment.

The presence of significantly different percentages of body fat in different experimental groups of hamsters after they have resumed regulating their body weight, may indicate that the weight regulation in the hamster does not involve lipostatic monitoring of fat reserves. This agrees well with the reduced nocturnal feeding rhythm in hamsters [27] in contrast to the highly nocturnal feeding pattern in the rat and the relative importance attributed to this rhythm in the lipostatic mechanism of weight regulation [16]. It also agrees well with the absence of estrogenic supression of body weight in the hamster [28] and the importance of this mechanism in the weight regulation of the female rat.

In the absence of a restraining influence of gonadal estrogen on growth and accumulation of body fat in the hamster, one may hypothesize that the rate and duration of its adolescent growth spurt largely determine the level around which its adult body weight is regulated.

If direct evidence substantiates the hypothesis that disc running stimulates growth hormone release then it may be profitable to examine the possible role of this hormone in normal weight regulation. Increased weight gain, growth, and hyperphagia in hamsters running on the disc exercisers may represent an exaggerated endocrine and behavioral response to an unnatural and artificial experimental situation. It may not be a coincidence that increased growth hormone release occurs during exposure to cold, during starvation, and in exericse [8]. All of these circumstances lead to energy expenditure and weight loss. A mechanism controlling growth hormone release commensurate to breakdown of body structure or to depletion of energy reserves would account well for the observed compensatory hyperphagia and increased weight gain following all of the mentioned circumstances of energy expenditure.

Comparative studies of feeding and weight-regulating mechanisms of a few species other than the rat have uncovered some dissimilarities in the regulatory capacities and response strategies employed in the defense of energy balance. Rat and hamster eat for calories and compensate well for the weight loss by starvation, but the guinea pig apparently does not [10]. Herbivorous hamster and guinea pig distribute their meals evenly over a $24 \mathrm{hr}$ period while rat confines most of its eating to the dark part of daily cycle. Yet all three manage to maintain their adult body weight stable over long periods of time. It may be of value to establish which features of these different weight-regulatory mechanisms represent ecological specializations and which are shared by mammals in general and man in particular.

\section{REFERENCES}

1. Borer, K. T., J. B. Powers, S. S. Winans and E. S. Valenstein. The influence of olfactory bulb removal on ingestive behaviors, activity levels, and self-stimulation in hamsters. J. comp. physiol. Psychol. 1974 (in press).

2. Carlisle, H. J. and E. Stellar. Caloric regulation and food preference in normal, hyperphagic, and aphagic rats. J. comp. physiol. Psychol. 69: 107-114, 1969.

3. Collier, G. H. Work: a weak reinforcer. Trans. N. Y. Acad. Sci. 32: $557-576,1970$.

4. Collier, G., A. I. Leshner and R. L. Squibb. Dietary selfselection in active and non-active rats. Physiol. Behav. 4: $79-82,1969$.

5. Cohn, C. and D. Joseph. Influence of body weight and body fat on appetite of "normal" lean and obese rats. Yale J. Biol. Med. 34: 598-607, 1962.

6. Earl, F. R., C. H. Vanetten, T. F. Clark and I. A. Wolff. Compositional data on sunflower seed. J. amer. oil. Chem. Soc. 45: $876-879,1968$

7. Frantz, A. G. and M. T. Rabkin. Estrogen stimulation of growth hormone release. J. clin. Invest. 44: 1048-1049, 1965 (Abstract).

8. Glick, S. M., J. Roth, R. S. Yalow and S. A. Berson. The regulation of growth hormone secretion. Recent Prog. Horm. Res. 21: $241-283,1965$.

9. Hervey, G. R. Regulation of energy balance. Nature 222: 629-631, 1969.

10. Hirsch, E. Some determinants of intake and patterns of feeding in the guinea pig. Physiol. Behav. 11: 687-704, 1973.

11. Hoffman, R. A., P. F. Robinson and H. Magalhaes. The Golden Hamster: Its Biology and Use in Medical Research. Ames: The Iowa University Press, 1968

12. Hunter, W. M. and F. C. Greenwood. Studies on the secretion of human-pituitary-growth hormone. Br. med. J. 1:804-807, 1964.

13. Jackinovich, W. Unpublished data. Department of Zoology, University of Michigan, Ann Arbor.

14. Kennedy, G. C. The role of depot fat in the hypothalamic control of food intake in the rat. Proc. R. Soc. Lond. B 140: $578-592,1953$.
15. Kodama, A. M. In vivo and in vitro determinations of body fat and body water in the hamster. J. appl. Physiol.. 31: 218-222, 1971.

16. Le Magnen, J., M. Devos, J. -P. Gaudilliere, J. Louis-Sylvestre and S. Tallon. Role of a lipostatic mechanism in regulation by feeding of energy balance in rats. J. comp. physiol. Psychol. 84: $1-23,1973$.

17. Mikolajzcak, K. L., C. R. Smith, Jr. and I. W. Wolff. Phenolic and sugar components of armavirec variety sunflower (Heli. anthus anuus) seed meal. J. agricult. Food Chem. 18: 27-32, 1970.

18. Pace, N. and E. N. Rathbun. Studies on body composition. III The body water and chemically combined nitrogen content in relation to fat content. J. biol. Chem. 158: 685-691, 1945 .

19. Powell, G. F., J. A. Brasel and R. M. Blizzard. Emotional deprivation and growth retardation stimulating idiopathic hypopituitarism. I. Clinical evaluation of the syndrome. New Engl. J. Med. 276: 1271-1278, 1967.

20. Powell, G. F., J. A. Brasel and R. M. Blizzard. Emotional deprivation and growth retardation stimulating idiopathic hypopituitarism. II. Endocrine evaluation of the syndrome. New Engl. J. Med. 276: 1279-1283, 1967.

21. Ruegamer, W. R., L. Bernstein and J. D. Benjamin. Growth, food utilization and thyroid activity in the albino rat as a function of extra handling. Science 120: $184-185,1954$.

22. Schalch, D. S. The influence of physical stress and exercise on growth hormone and insulin secretion in man. J. Lab. clin. Med. 69: 256-269, 1967 .

23. Teitelbaum, P. Sensory control of hypothalamic hyperphagia. J. comp. physiol. Psychol. 48: 156-163, 1955.

24. Wade, R. N. and I. Zucker. Hormonal and developmental influences on rat saccharin preferences. J. comp. physiol. Psychol. 69: $291-300,1969$

25. Widdowson, E. M. and R. A. McCance. The effect of finite periods of undernutrition at different ages on the composition and subsequent development of the rat. Proc. R. Soc. Lond. $B$ 158: $329-342,1963$. 
26. Zucker, I. Body weight and age as factors determining estrogen responsiveness in the rat feeding system. Behav. Biol. 7: $527-542,1972$.

27. Zucker, I. and F. K. Stephan. Light-dark thythms in hamster eating, drinking and locomotor behavior. Physiol. Behav. 11: $239-250,1973$.

28. Zucker, I., G. N. Wade and R. Ziegler. Sexual and hormonal influences on eating, taste preferences and body weight of hamsters. Physiol. Behav. 8: 101-112, 1972. 